\title{
Paleoambientes de la Cornisa Cantábrica y su relación con el desarrollo del arte del Paleolítico Superior *
}

\author{
J. GonzÁlez EChegaray **
}

No cabe duda de que puede existir una concatenación entre los distintos periodos climáticos del Würm superior en la Europa occidental y las diversas fases cronológicas del arte rupestre paleolítico. Ello se debe al hecho de que circunstancialmente las condiciones climáticas cambiantes han podido determinar la habitabilidad o no de algunas cuevas con figuras rupestres en distintos momentos, lo que supone un criterio, externo pero seguro, de datación cronológica de ciertos conjuntos artisticos.

Tal sería el caso de hundimientos en la boca de las cuevas, o en anegamiento por las corrientes subterráneas de las galerias de acceso a las salas pintadas, y otras contingencias que impedirian la entrada alli del hombre paleolitico durante largas etapas, o el desprendimiento de lajas del techo pintado debido a factores climáticos, o la formación de sedimentos recubriendo figuras parietales. Todas estas contingencias se dan en las cuevas pintadas y han servido a veces como criterios de fijación cronológica por los autores clásicos como $\mathrm{H}$. Breuil y A. Leroi-Gourhan. Sin embargo, según ya hemos dicho, se trata de circunstancias excepcionales, que, aunque valiosas en casos-limite, no permiten montar una sólida teoría, bien contrastada, sobre la cronología del arte rupestre en sus distintas etapas y estilos.

No obstante, en otros aspectos, las variaciones climáticas del Pleistoceno final pueden arrojar mucha luz sobre el problema de la cronologia

* Lección pronunciada en el curso “Introducción al arte rupestre prehistórico de la Peninsula Ibérica». Ávila, 9-12 de julio de 1991.

** Instituto de Investigaciones Prehistóricas (Chicago-Santander). 
del arte rupestre paleolítico. Se trata de la incidencia de los cambios climáticos sobre la fauna, $y$, dado que los animales constituyen uno de los motivos más importantes del arte paleolitico, la fauna representada en un momento dado puede indicarnos el tipo de clima entonces existente. $Y$, puesto que tenemos un conocimiento genérico de la época a que pertenecen las representaciones, se tratará de buscar en los esquemas climáticos de entonces el momento preciso que corresponda al medio ambiente reflejado por los animales pintados o grabados. Asi podrian datarse con alguna precisión, cuando las circunstancias lo permitieran, diversos conjuntos de arte rupestre paleolitico. La comparación de varios de ellos entre si permitiria a su vez montar una teoría cronológica coherente sobre la cronología y evolución del arte paleolítico.

Aunque es un tema que ha sido tratado y discutido en teoria, no ha solido ser sistemáticamente aplicado al estudio del arte rupestre. Por nuestra parte, hemos venido insistiendo en su importancia repetidas veces desde hace veinticinco años (González ECHEgaray 1966), habiendo realizado ensayos de aplicación a casos concretos como la Cueva de las Chimeneas (Gonzalez Echegaray 1974) y la del Castillo (González EcheGaRAY 1972). En esta misma linea se ha colocado E. Ripoll por lo que respecta al análisis de la Cueva de las Monedas (RIPOLL 1972). También Altuna ha realizado análisis de este tipo en Altxerri (ALTUNA-APELLANIZ 1976) y en Ekain (ALTUNA-APELLANIZ 1978), aunque insiste mucho en que hay que tratarlo con toda reserva (ALTUNA 1984), y Moure lo ha desarrollado en el caso de la Cueva de Tito Bustillo (Moure 1980; Balbin y Moure 1982). El tema ha sido objeto de un estudio de conjunto por parte de $A$. Castaño (1987), aplicándolo a varias cuevas del norte de España. A pesar de esto, creemos vale la pena volver ahora sobre ello, ya que por una parte brinda aún, profundizando en él, muchas posibilidades, mientras que, por otra, deben despejarse ciertas dificultades que aún subsisten acerca del valor del método en si.

Al margen de cualquier interpretación del significado del arte rupestre, damos aqui por sentado que la fauna representada refleja el medio ambiente climatológico del momento en que se realizaron las obras de arte. Pero en primer lugar, esto no tiene por qué ser necesariamente asi, mientras las especies representadas no sean rigurosamente sensibles a determinados climas, pues, como todos sabemos, hay algunos mamiferos que se muestran prácticamente indiferentes a los cambios climáticos. En segundo lugar, cabe plantearse el interrogante de si los artistas paleoliticos trataron de reflejar el mundo animal realmente existente en el momento, o, más bien, aquellas especies deseadas y que precisamente resultaban cada día más escasas a causa de los cambios climáticos que se 
iban produciendo. Todo depende de las intenciones que supongamos en el artista.

Por de pronto, es cierto que no existe una adecuación exacta entre la fauna recogida en los yacimientos de cuevas pintadas en las que pinturas y habitación se supone que fueron rigurosamente contemporáneos. En el Magdaleniense de Altamira la especie mejor representada, según Klein, es el ciervo con un $62 \%$, seguida del bisonte con un $8,3 \%$ y el caballo con un 4,2\% (Freeman, Gonzalez Echegaray, Klein y Crowe 1988: 29). En las representaciones artísticas que se estiman contemporáneas, es decir, en el conjunto más reciente del techo la proporción seria casi la inversa con un $79 \%$ de bisontes, un $14,7 \%$ de ciervos y un $5,9 \%$ de caballos (GonzAlez Echegaray 1986: 26-27). Lo mismo sucede en Ekain, según Altuna (ALTUNA y APELLANIZ 1978: 106-107), donde el yacimiento da para el Magdaleniense más antiguo el $87,8 \%$ de ciervos, el $8,1 \%$ de cabras, el $1,3 \%$ de bisontes y sólo el $0,6 \%$ de caballos, en tanto que las representaciones tienen un valor exactamente inverso con $57,6 \% ; 18,6 \% ; 8,5 \%$ y $5,1 \%$ respectivamente.

Pero todos estos datos y consideraciones precisan de una cuidada matización, que lejos de desposeer el valor al argumento, le devuelven todo su vigor, siempre que se trate con la debida cautela y, sobre todo, con sentido común.

Ante todo, hay que asegurarse de la contemporaneidad del conjunto de figuras que se deben analizar, para lo cual es preciso recurrir a criterios artísticos de uniformidad de estilo y de técnicas, e indicios de que el conjunto responde a un plan en lo que respecta a la selección y situación de las figuras en la galeria de la cueva. A estos criterios podrian añadirse otros complementarios de carácter geológico sobre las posibilidades y limitaciones de acceso a la galería o cueva en cuestión, y a los que ya hemos aludido anteriormente. Conviene, no obstante, señalar que la contemporaneidad de que hablamos, no debe tomarse necesariamente en un sentido estricto, como si las pinturas hubieran sido realizadas todas de una vez y el mismo día. Basta una contemporaneidad relativa, que se trate de un solo periodo, aunque entre la ejecución de unas y otras figuras hayan pasado varios años y hasta generaciones. En el caso de una iglesia de un estilo definido, románico por ejemplo, en donde decimos que todas las representaciones (pinturas o esculturas) pertenecen al mismo periodo, aunque la iglesia tardara en construirse cien años. Pero no es éste el caso de una catedral, en la que existen construcciones de distintos estilos y épocas (románico, gótico, renacentista...), donde sólo cada una de éstas puede considerarse contemporánea, pero no todo el edificio. 
Una vez que tengamos el convencimiento moral de que se trata de un conjunto homogéneo, sea que ocupe en exclusiva toda la cueva o simplemente una o varias galerias, compartiendo la cueva con otros conjuntos independientes, es preciso estudiar la fauna representada desde el punto de vista ecológico. Para ello, no sólo hay que atender a especies que directamente son significativas climáticamente, como puede ser el reno, que sólo existe en la región cantábrica durante las etapas más frias, sino tambièn a otras especies que acusan biotopos definidos, como el ciervo con relación al bosque o los caballos en relación a las praderas. A través de otros estudios, por ejemplo la palinología, conocemos el grado de desforestación del paisaje cantábrico en los distintos periodos del Würm superior, lo que puede ofrecernos una pista para ubicar el momento en que se realizan las figuras estudiadas.

Desde luego, cuando se trata de animales muy sensibles al clima, como el reno, el saiga, el alce, el mamut, el rinoceronte lanudo, el glotón y la liebre ártica - por referirnos ahora a las representaciones que han sido identificadas entre las figuras rupestres de la zona cantábrica-, no hay duda de que su presencia, aunque sólo esté representada por una figura, siempre que su identificación sea segura, es una prueba por si misma de que el conjunto ha sido ejecutado en un momento de clima muy frio. No es sostenible seriamente que los hombres del Paleolitico dibujaran realisticamente un animal que no habian visto nunca, aunque hipotéticamente tuvieran noticia de su existencia por relatos de sus antepasados, trasmitidos por tradición, o por contactos con otros grupos humanos establecidos en lejanas regiones donde todavia subsistian tales especies.

Sin embargo, cuando se trata de especies más indiferentes al clima, aunque acusen biotopos especiales, resulta necesario atender al número de animales representados. En una región de donde han desaparecido los grandes bosques, pueden aún subsistir algunos ciervos refugiados en zonas marginales de bosque-galeria o de monte bajo o adaptados a nuevas condiciones *. Por tanto, la presencia de un solo ciervo no significa que se trate necesariamente de un periodo de clima benigno en el que tuvo su máximo desarrollo en bosque mixto. En cambio, si en un conjunto de

* Según nos comenta L. G. Freeman, el Cervus elaphus, que pasta en las praderas junto a los bosques y se oculta en la espesura, a pesar de ser una especie propia del ecosistema llamado "parque" (mezcla de bosque y praderas), puede adaptarse a terrenos totalmente despejados, como de hecho sucede en Escocia. Sin embargo, no es presumible que esta adaptación histórica hubiera tenido lugar en "condiciones naturales" normales, ya que se ha realizado en territorios, donde el ciervo carecia de la presión depredadora de otras especies (singularmente el hombre) y donde, por tanto, no tenia necesidad de refugiarse en la espesura. 
figuras, en donde hay distintas especies representadas, predominan los ciervos, puede considerarse como un indicio serio de que aquellas pinturas fueron ejecutadas en un interestadio de clima templado, en donde el ambiente forestal dominaba sobre la pradera, puesto que parece dificil pensar que el cazador paleolitico, que está representando todos los grandes herbivoros que ve, insista de modo especial en dibujar reiterada y detalladamente aquellos con cuya silueta y costumbres está menos familiarizado. En los dibujos infantiles de coches que hacen nuestros niños, abundan más la representación de marcas conocidas en el mercado, que son los coches que el niño ve y con los que está familiarizado, que no los ejemplares raros, aunque la alusión esporádica a éstos no pueda descartarse de forma absoluta.

En nuestra opinión, la fauna representada en un conjunto rupestre homogéneo (queremos decir, contemporáneo) ha de reflejar en sus lineas generales el mundo de los grandes herbivoros del momento, no sólo cualitativa, sino cuantitativamente (tomando esta última acepción en un sentido amplio, sin pretender ir más lejos de lo que el buen sentido puede aconsejarnos). Contrasta parcialmente esta toma de postura con los resultados del estudio de la fauna en los yacimientos, según ya hemos indicado. A este respecto hay que recordar, en primer lugar, el hecho de que, para manejar datos válidos, es imprescindible seleccionar yacimientos directamente relacionados con las figuras rupestres, huyendo de generalizaciones y aproximaciones que desembocan en abusos del método. Pero incluso, una vez confirmada razonablemente la contemporaneidad entre conjuntos rupestres y yacimientos, en contra del proceder habitual, creemos debe darse preferencia, en orden a interpretar las condiciones medioambientales del momento, a las representaciones pictóricas más que a los restos de alimentación que constituyen esencialmente la fauna de un yacimiento.

Es cierto que los huesos hallados en un yacimiento se refieren a animales indudablemente existentes en aquella época, pero la proporción númerica existente entre unas especies y otras no tiene por qué obedecer a la realidad ecológica del momento, ya que aqui interviene un factor decisivo, que es la preferencia humana y la selección de recursos en orden a la alimentación. La abundancia de ciervos en un yacimiento se debe a que era la especie más cazada por el hombre en aquel momento, pero no quiere decir que fuera la especie más abundante entre los grandes mamiferos, sino la que reunia mejores condiciones de captura y presentaba un mayor rendimiento económico. Por tanto, sería abusivo afirmar que el paisaje estaba constituido por un bosque interminable. En realidad no puede destacarse que hubiera grandes extensiones de pradera y que por ella pulularan otros herbivoros, como los bisontes, pero que estos no 
fueran habitualmente cazados, debido a las dificultades y peligros que ello conllevaba. Los hombres del Paleolitico final en la costa cantábrica prefirieron especializarse en la captura del ciervo, que además proporcionaba materia prima muy aprovechable para manufacturas, como el asta con destino a ciertos utensilios y una piel fina, resistente y de proporciones adecuadas para el vestido humano.

Tampoco puede descartarse que la caza eventual del bisonte - continuando con el mismo ejemplo - fuera seguida de un descuartizamiento de la pieza in situ, para proveerse de la carne y no ir cargado a la cueva con la pesada osamenta, tal y como hacian algunos indios americanos de las praderas. En cambio, cuando se trata de un cérvido, éste puede ser echado al hombro y llevado en su integridad al yacimiento para ser aquí descuartizado y debidamente aprovechado. Resulta así que el hecho de que los huesos recogidos en un yacimiento paleolítico se refieran en su mayoría a cérvidos y que, aunque no falten, sean escasos los de bóvidos y otros grandes mamiferos, no supone necesariamente que el medio ambiental fuera más propicio al desarrollo de aquellas especies, que al de éstas, sino que obedece a un factor de carácter esencialmente económico. Asi es posible que el conjunto de representaciones rupestres pueda en determinados casos darnos una visión más realista del medio ambiente, que la misma fauna de un yacimiento. Todo esto independientemente de la intencionalidad y significado del arte rupestre, esté o no relacionado con la magia de caza o con otras ideas religiosas y sociales.

Realizadas todas estas consideraciones y procediendo siempre con cautela y las debidas reservas pensamos que el estudio del arte rupestre puede contribuir al mejor conocimiento del mundo animal de finales del Pleistoceno (RIPOl 1961; Blanchard 1964; Band 1966; Madariaga 1969; LIÓN 1971; ALTUNA y APELLÁNIZ 1978) y consiguientemente de una reconstrucción ecológica del momento. $Y$ además puede ofrecer datos objetivos que ayuden a su relativa autodatación, por el procedimiento que aqui venimos desarrollando.

Dado que la época en que se desarrolla casi en su integridad el arte rupestre paleolítico corresponde a los periodos Solutiense y Magdaleniense, lo que ya nadie pone en duda después de la revisión de LeroiGourhan, debemos ahora exponer muy brevemente las conclusiones a que han llegado los especialistas acerca del clima en la costa cantábrica durante la etapa que transcurre entre los años 20.000 y $10.000 \mathrm{BP}$ y que coincide con el llamado Würm IV.

El tema ya ha sido tocado por nosotros en otras ocasiones (GonZÁLEZ ECHEGARAY 1966, 1972-73 y 1975) y recientemente ha sido vuelto a consi- 
derar por González Sainz (1989) y por L. Straus (1991). Ni qué decir tiene que estos intentos de síntesis se fundan en estudios puntuales de tipo geológico como los de Obermaier, Vega de Sella, Hernández Pacheco, Butzer, Hoyos, Laville..., palinológicos como los de Arl. Leroi-Gourhan, Boyer-Klein.., o zoológicos como los de Altuna, Klein, Madariaga..., etc., cuyas numerosas monografias no podemos citar aquí.

Hay una etapa templada entre el Würm III y el Würm IV, que corresponde fundamentalmente a los interestadios de Laugerie y Lascaux, en torno al 20.000 y $16.500 \mathrm{BP}$, la cual se halla flanqueada por sendas fases estadiales frias: el fina del Würm Illb hacia el $22.000 \mathrm{BP}$ y el comienzo del Würm IV (Dryas I) hacia el $16.000 \mathrm{BP}$, y está interrumpida por un breve estadio intermedio hacia el 18.500 . Toda esta larga etapa desde el final del Würm IIIb hasta muy entrado el interestadio de Lascaux coincide en el Norte de España con el desarrollo de la cultura Solutrense.

El Solutrense cantábrico, bien estratificado en la Cueva de Las Caldas (CoRchon 1981), tiene, pues, una primera etapa fria que acaso coincida con el estrato $10 \mathrm{del}$ Castillo. Cuenta después con un periodo templado, que puede estar bien representado en Cueto de la Mina, Bolinkoba y Ermitya. Presenta a continuación otro momento frío que se aprecia asimismo en la Riera, Morín, Altamira y Aitzbitarte IV. Finalmente muestra una última etapa templada - ya contemporánea de los primeros Magdalenienses en Francia-, que está igualmente presente en la Riera (StRaus y CLARK 1986).

Tenemos a continuación el complejo mundo del Magdaleniense, que se desarrolla desde el Interestadio de Lascaux hasta la oscilación suave de Alleröd. Corresponde en sus lineas generales al Würm IV y va desde el 16.000 al $10.000 \mathrm{BP}$.

Comprende, pues, teóricamente cinco etapas templadas y cuatro frias, según el siguiente orden: Lascaux, Dryas la, Angles, Dryas lb, Pre-Bölling, Dryas Ic, Bölling, Dryas II y Alleröd. En la práctica esta secuencia no existe completa en ningún yacimiento de la Cornisa Cantábrica, por lo que se precisa recurrir a una simplificación, que probablemente se ajusta más a la realidad no sólo de nuestras fuentes de información, sino también a los hechos. Naturalmente esto no quiere decir que no existan multitud de variaciones y matizaciones en el clima (no sólo en cuanto a temperatura se refiere, sino principalmente respecto a la humedad) a lo largo de toda la etapa, que en cierta forma hace más compleja cualquier visión de conjunto, como evidencian las estratigrafias parciales de nuestros yacimientos, pero no puede hablarse claramente de tantos periodos "fríos" o "templados". Ante estas circunstancias, M. Hoyos ha creado su propia terminologia para la zona, con independencia de las fases europeas de 
nombre ya consagrado, que denomina Würm IV Cantábrico I-IX, etapas que abarcan desde el estudio post-Laugerie hasta el Dryas III (LAVILLE y Hoyos 1981).

En todo caso, está bien documentada la presencia del Magdaleniense Inferior Cantábrico (Magdaleniense III) en el interestadio de Lascaux hacia el 16.500 BP y su prologanción durante el Dryas la (Rascaño, Riera, Ekain) hacia el $16.000 \mathrm{BP}$. También su presencia durante un ulterior interestadio templado, probablemente el de Angles (Rascaño y Riera) hacia el 15.200 BP. A juzgar por las fecha de C-14 y ciertos indicios climáticos, Altamira y Erralla podrian ser atribuidas al Dryas la.

A partir de aqui la serie deja de ser segura. Aunque el Magdaleniense Inferior se prolonga hasta fechas relativamente avanzadas en el Juyo, en otros lugares parece iniciarse el Magdaleniense Superior, como en Tito Bustillo en un clima frio con una datación en torno al $14.250 \mathrm{BP}$, lo que deberia corresponder en principio al Dryas IC (Moure y CANo 1976). Parece aún insegura la atribución real y la ubicación exacta de un Magdaleniense Medio, que estaría representado en algunos yacimientos asturianos (La Paloma, Las Caldas, Cueto de la Mina, La Viña...), asi como en los yacimientos vizcainos de Santimamiñe y Ermitya. En Las Caldas corresponde a una etapa fria, sin que se pueda determinar con seguridad si se trata en efecto del Dryas Ib.

La secuencia del Magdaleniense Superior aparece relativamente bien estratificada en Rascaño, en donde un hiatus de carácter erosivo podría aludir al Bölling, mientras que el nivel inmediatamente superior, con un Magdaleniense desarrollado en un clima que comienza a enfriarse en torno al $12.800 \mathrm{BP}$, podria coincidir con el fin de ese interestadio y el inicio del Dryas II. Hacia el 12.280 aún sigue, con variaciones, un clima frio y húmedo. Otra discordancia erosiva debe corresponder al Alleröd, en tanto que el Aziliense local se desarrolla en una etapa fría atribuida al Dryas III en torno al $10.500 \mathrm{BP}$. La presencia aún del Magdaleniense final durante la oscilación suave de Alleröd parece estar documentada en Erralla y Ekain (Altuna, Baldeon y Marriezcurrena 1985; Altuna y Merino 1984).

Como puede comprobarse, hay demasiados cambios climáticos importantes durante los 10.000 años de desarrollo principal del Arte Rupestre cantábrico como para poder clasificar y encajar cronológicamente con precisión un conjunto faunistico de los que aparecen representados en las cuevas cantábricas. La existencia de un clima frio, por ejemplo, puede atribuirse a periodos muy distintos, incluso sin salirse del ámbito Solutreo-Magdaleniense.

Asi pues, para que el argumento del medio ambiente pueda constituirse en un criterio útil y fiable de datación, es necesario combinarle con 
los demás procedimientos utilizados en la elaboración de un esquema secuencial del arte paleolítico, principalmente los criterios estilisticos. Sólo asi el argumento ambiental puede constituirse en una ayuda apreciable.

Vamos ahora a presentar algunos ejemplos que puedan ilustrar el alcance de todo cuanto aquí hemos expuesto de una forma más bien teórica.

En primer lugar, nos referiremos a la Cueva del Castillo, que presenta, como se sabe, una suma de conjuntos pictóricos de distintas épocas, formando tres santuarios (LERol-GouRHAN 1965). De acuerdo con nuestro análisis (GonzÁlEZ ECHEGARAy 1972), el santuario más antiguo del llamado "Estilo III» presenta una fauna de ambiente estadial con predominio de animales de estepa (bisontes, caballos) y con una representación de lo que probablemente es un mamut. Desde el punto de vista estilístico, es casi seguro que debe atribuirse a un momento avanzado del Solutrense. Ahora bien, en el Solutrense Superior tuvo lugar el estadio Inter LaugerieLascaux, entre el 19.000 y el $18.000 \mathrm{BP}$, al que razonablemente habria que remontar el conjunto de figuras en cuestión. El segundo santuario pertenece al "Estilo IV antiguo" y ofrece un conjunto faunistico en el que tienden a cobrar más importancia las figuras de cérvidos y cápridos, acusando al parecer un clima más benigno. Siendo cronológica y estilisticamente más reciente, podría ser atribuido al interestadio de Lascaux, que en el norte de España corresponde al fin del Solutrense y comienzos del Magdaleniense Inferior (entre el 18.000 y el 16.500 BP). Hay un tercer santuario, donde al parecer se acusa un recrudecimiento del clima, también perteneciente al "Estilo IV antiguo" aunque algo más reciente que el anterior. Podria relacionarse con el Dryas la, dentro aún del Magdaleniense Inferior Cantábrico, hacia los años 16.500-15.500 BP. Por su parte, en el yacimiento de la cueva este período está ampliamente representado, con fauna más bien fria, entre la que se llega a citar incluso al reno (CABRERA 1984: 354-356).

La Cueva de las Chimeneas, que carece de yacimiento propio y que sin duda constituye toda ella un santuario único, debió ser decorada durante un interestadio templado a juzgar por la fauna, en la que abundan los cérvidos y cápridos, son escasos los caballos y los uros y faltan los bisontes y los renos. Dados sus rasgos estilisticos, las figuras parecen contemporáneas del santuario intermedio del Castillo y podrian remontarse o a las últimas etapas del Solutrense regional, o a los comienzos del Magdaleniense Superior, es decir, al interestadio de Lascaux. 
Con las Chimeneas contrasta la vecina Cueva de las Monedas, donde las pinturas, aún siendo igualmente de trazo negro, presentan un estilo más evolucionado, en el que no faltan los moldeados o la cuidadosa terminación de ciertas partes del cuerpo, sobre todo las patas. La fauna representada es muy distinta, ya que en ella figura el reno y abundan el caballo y el bisonte. No cabe duda, que debe ser atribuida a una fase ulterior ya en pleno Magdaleniense Superior, quizás al Dryas Ic, si no es, como parece más probable al Dryas II.

Por lo que se refiere a Altamira, el tema merece una especial atención, dada la importancia de esta cueva y el control de relación entre fauna representada y yacimiento. Éste, como hemos dicho, presenta dos niveles, Solutrense Superior y Magdaleniense Inferior, y después de éste se halla atestiguado el desplome del techo y taponamiento de la entrada a la cueva, lo que haría muy dificil el eventual acceso al interior por parte de unos hombres que ya ciertamente no vivian en ella (BrEUIL y OBERmaier 1935; Gonzalez Echegaray 1988; Freeman 1988).

Leroi-Gourhan distingue, al menos, tres fases en la realización del conjunto pictórico de la cueva: lo que él llama "la serie negra», que se extiende a lo largo de todas las galerias y que, además de pinturas negras, incluye también grabados. Ésta pertenecería al Estilo III, es decir, al Solutrense final de la Costa Cantábrica. La segunda fase, representada por el "Gran techo policromo» de Altamira, correspondería al estilo IVa, es decir, al Magdaleniense lll cantábrico. Aún existiria un tercer momento en el Magdaleniense Superior al que se atribuirian ciertos grabados de la llamada "Cola de caballo" o galería final de la cueva. Por distintas razones, como la unidad temática que se descubre entre esta galería y el famoso techo (FREEMAN et alii 1987) y por el mismo hecho citado, de que la cueva no debió ser visitada después de la realización de aquél, pensamos que ambos conjuntos corresponden a un solo momento. En cambio, en el gran techo hay claramente dos paneles bien distintos y de cronologia diferente como ya expuso en su día Breuil (BREull y OBERMaIER 1935). Uno es el más conocido y situado a la izquierda, constituido por las figuras «polícromas» de 27 bisontes, tres ciervos y dos caballos; y el otro a la derecha, por figuras rojas o negras que representan 15 caballos, tres bisontes, tres ciervos, un alce, un cáprido y un uro. Este último conjunto es evidentemente más antiguo que el anterior. La presencia del alce y la abundancia de caballos quizá sugiera un clima más riguroso que el otro conjunto, si bien este último, en donde el bisonte constituye la especie protagonista, tampoco parece aludir precisamente a un momento de clima beningno. De acuerdo con estas caracteristicas, cabría pensar que el conjunto de las figuras monócromas fue ejecutado en el estadio Inter Laugerie-Lascaux, durante el Solutrense Superior, que aparece bien es- 
tratificado en el yacimiento de la cueva y presenta una fauna al parecer fría. Por el contrario, el conjunto de policromos o bicromos sería realizado con posterioridad, al final del interestadio de Lascaux, cuando tal vez comenzaban los primeros sintomas del Dryas la, lo que corresponde al nivel de ocupación más moderno del yacimiento que es, según ya hemos dicho, un Magdaleniense Inferior Cantábrico. Esta atribución de los bisontes policromos al Magdaleniense III fue defendida en su dia por Leroi-Gourhan (Lerol-Gourhan 1965), contra el abate Breuil que suponia que tales figuras fueron pintadas en el Magdaleniense Superior (BREUIL y OBERMAIER 1935). Nada diremos ahora respecto a las figuras de la "Serie negra", que probablemente se distribuyan entre las dos etapas señaladas en el gran techo, pero cuyo estudio requiere ulteriores análisis que todavía no hemos concluido.

Otro ejemplo podría ser la cueva de Ekain. El conjunto parece representar un ambiente de transición de un clima templado a otro frio, donde abundan los animales de estepa (caballo, bisonte... y quizá rinoceronte). Dado que estilísticamente el conjunto de Ekain puede compararse con Altamira, su atribución al Magdaleniense Inferior Cantábrico parece justificable, tanto más cuanto que el yacimiento de la cueva ofrece un importante nivel de ocupación de esa época (nivel VII). Precisamente en él se comprueba el tránsito de un interestadio templado (Lascaux) a un estadio frio (Dryas la).

Señalemos ahora el caso de Tito Bustillo, donde en el conjunto de figuras representadas abunda el caballo, seguido por el ciervo, la cabra y los grandes bóvidos, pero donde es altamente significativa la presencia del reno (por lo menos siete figuras), indicando evidentemente un ambiente frio (BALBIN y MOURE 1982). Las pinturas pertenecen estilisticamente a un momento más evolucionado. En el yacimiento de la cueva se halla atestiguado un nivel de comienzos del Magdaleniense Superior y de clima frio, atribuible al Dryas lc, que puede corresponder al ambiente reflejado por el conjunto artístico.

Por su parte, Altxerri, con una fauna fria, donde predominan las representaciones de bisonte y reno y aparece además el saiga, el rebeco y probablemente el glotón, el zorro polar y la liebre ártica, representa un momento álgido en el Magdaleniense Superior, que, dados además sus caracteres estilisticos, probablemente deba atribuirse al Dryas II, siendo contemporáneo de las Monedas.

Este es el estado de la cuestión al momento presente, tal y como nostros lo vemos. No puede, a nuestro juicio, rechazarse sin más el «argumento ecológico» cuando llega el momento de estudiar y tratar de datar las pinturas y grabados paleoliticos de una cueva. Más aun, este método 
no aislado sino junto con los demás, puede permitirnos precisiones nada despreciables que nos dejen comprender y encajar el arte rupestre. Pero sería temerario ir más lejos y utilizarlo irresponsablemente, como si se tratara de algo seguro e incontrovertible. La prudencia y la mesura son siempre en Prehistoria, pero de forma especial en este caso, absolutamente imprescindibles.

\begin{tabular}{|c|c|c|c|c|}
\hline CRONOLOGIA & FASES & CLIMA & CULTURAS & ARTE RUPESTRE \\
\hline 10.400 & Dryas III & Fresco & & \\
\hline 11.000 & Alleröd & Templado & Aziliense & \\
\hline \multirow[t]{2}{*}{12.300} & Dryas 11 & Frio & & $\begin{array}{l}\text { Monedas y Altxe- } \\
\text { rri }\end{array}$ \\
\hline & & & Magdaleniense & \\
\hline 13.000 & Bölling & Templado & Superior & \\
\hline 14.000 & Dryas Ic & Frio & & Tito Bustillo \\
\hline 14.300 & Pre-Bölling & Templado & & \\
\hline & & & Magdaleniense & \\
\hline 14.800 & Dryas $1 \mathrm{~b}$ & Frio & Medio & \\
\hline 15.200 & Angles & Templado & & \\
\hline 15.900 & Dryas la & Frio & $\begin{array}{l}\text { Magdaleniense } \\
\text { Inferior }\end{array}$ & $\begin{array}{l}\text { Castillo III, Altami- } \\
\text { ra II y Ekain }\end{array}$ \\
\hline 16.500 & Lascaux & Templado & & $\begin{array}{l}\text { Castillo II y Chi- } \\
\text { meneas }\end{array}$ \\
\hline 18.000 & & & $\begin{array}{l}\text { Solutrense } \\
\text { Final }\end{array}$ & \\
\hline 18.500 & $\begin{array}{l}\text { Inter Laugerie- } \\
\text { Lascaux }\end{array}$ & Frio & $\begin{array}{l}\text { Solutrense } \\
\text { Superior }\end{array}$ & $\begin{array}{l}\text { Castillo I y Altami- } \\
\text { ra I }\end{array}$ \\
\hline
\end{tabular}

Ensayo de una posible ubicación cronológica de algunos conjuntos rupestres de la Cornisa Cantábrica.

\section{BIBLIOGRAFIA}

Altuna, J., y Apellaniz, J. M., 1976: “Las figuras rupestres paleoliticas de la Cueva d'Altxerri (Guipuzcoa)", Munibe, 28, 1-242.

- 1978: "Las figuras rupestres de la Cueva de Ekain (Deva, Guipúzcoa)", Munibe, 30, 151.

Altuna, J; Baldeon, A., y Mariezcurhena, C., 1985: "Cazadores magdalenienses en Erralla (Cestona, Pais Vasco)», Munibe, 37, 1-206.

Altuna, J., y Merino, J. M., 1984: El yacimiento prehistórico de la Cueva de Ekain (Deba, Guipuzcoa). San Sebastián, Sociedad de Estudios Vascos.

Balbin, R. y Moure, J. A., 1982; "El panel principal de la Cueva de Tito Bustillo (Ribadesella, Asturias)", Ars Praehistorica, 1, 47-97. 
BANDI, H.-G., 1968: “Art Quaternaire et zoologie». En RIPOLL, E. (ed.) (1968), Simposio Internacional de Arte Rupestre, Barcelona, 1966. Barcelona, 1968, 13-19.

BLANCHARD. J., 1964: «Informations recherchées d'aprés les équides européens figurés». En Pericot, L. y Ripoll, E. (eds.), Prehistoric art of the Western mediterranean and the Sahara, 3-34. Viking Fund Publications in Anthropology 39. Nueva York.

Breull, H., y Obermaier, H., 1935: The cave of Altamira. Madrid, Junta de las Cuevas de Altamira, Hispanic Society of America, y Academia de la Historia.

Cabrera, V., 1984: El yacimiento de la Cueva de "El Castillo" (Puente Viesgo, Santander). Madrid, Bibl. Praehist. Hispana, vol. XXII.

CAstano, A., 1977: "Aspectos ecológicos del arte parietal paleolitica en Cantabria", en Estudio de Arte paleolitico. Madrid, CIMA, 15, 7-66.

Corchón, S., 1981: Cueva de Las Caldas, San Juan de Priório (Oviedo). Madrid, Ministerio de Cultura

FreEman, L., 1988: "The stratigraphic sequence at Altamira, 1880-1991", Espacio, Tiempo y Forma (Homenaje a E. Ripoll), I, 149-163.

Freeman, L.; Gonzalez Echegaray, J.; Bernaldo de Quirós, F., y Ogden, J., 1987: Altamira Revisited. Santander, Instituto para Investigaciones Prehistóricas.

Freeman, L.: Gonzalez Echegaray, J.; Klein, R., y Crowe, W., 1988: "Dimensions of Reserarch at El Juyo". En Dibble, H., y Montet-White, A. (eds.), Upper Pleistocene Prehistory of Western Eurasia. Philadelphia, University of Philadelphia

Gonzalez Echegaray, J., 1966: "Sobre la cronología de la glaciación Würmiense en la Costa Cantábrica", Ampurias, 18, 1-12

- 1968: "Sobre la datación de los santuarios paleoliticos". En RIPOLL, E. (ed.), Simposio de Arte Rupestre Barcelona 1966, 61-65.

- 1972: "Notas para el estudio cronológico del arte rupestre de la Cueva del Castillo", en Santander Symposium, 409-419.

- 1972/73: “Consideraciones climáticas y ecológicas sobre el Magdaleniense III en el Norte de España", Zephyrus, 23-23, 167-187.

- 1974: Las pinturas y grabados de la Cueva de las Chimeneas. Barcelona, Monografías de Arte Rupestre, Arte Paleolítico 2.

- 1975: “Clima y ambiente durante el Paleolítico", en La Prehistoria en la Cornisa Cantábrica, 33-60.

- 1988: “El Magdaleniense de Altamira”, Espacio, Tiempo y Forma (Homenaje a E. Ripoll), I. 165-175.

Gonzalez Sainz, C., 1989: El Magdaleniense Superior/Final de la Región Cantábrica. Santander, Ed. Tantin.

Laville, H., y Horos, M., 1981: "Estudio geológico de la Cueva de Rascaño". En Gonzalez Echegaray, J., y Barandiaran, I., El Paleolitico Superior de la Cueva del Rascaño (Santander), 189-210.

Lerol-Gourhan, A., 1965: Préhistoire de l'Art Occidental. Paris, Mazenod.

Lión Valderrabano, R., 1971: El caballo en el arte cántabro-aquitano. Santander, Patronato de las Cuevas Prehistóricas de la Provincia de Santander.

Moure, J. A., 1980: Las Pinturas y Grabados de la Cueva de Tito Bustillo: Significado cronológico de las representaciones de animales. Valladolid, Studia Archaeologica 61

Moure, J. A., y CANO, M., 1966: Excavaciones en la Cueva de "Tito Bustillo". Trabajos de 1975. Oviedo.

Ripoll-Perello, E., 1961: «El problema de las representaciones de elefantes de piel desnuda en el arte cuaternario", en Bericht über den V. Int Kongress für Vor- un Frühgeschichte, 703-4.

- 1972: La Cueva de Las Monedas en Puente Viesgo (Santander). Monografias de Arte Rupestre, Arte Paleolitico, 1. Barcelona 
Straus, L., 1971: "Southwestern Europe at the last glacial maximum», Current Anthropology, 32, 189-199.

Straus, L., y Clark, G., 1986: La Riera Cave, Stone Age Hunter-Gatherer Adaptations in Northern Spain. Tempe, Arizona State University. 